\title{
Early lessons on assembling a center for bariatric endoscopy
}

\author{
Jack Mlabasati, Mohammad Bilal, Jonah Cohen \\ Center for Advanced Endoscopy, Beth Israel Deaconess Medical Center, Harvard Medical School, Boston, MA 02115, USA. \\ Correspondence to: Dr. Jonah Cohen, Assistant Professor of Medicine, Director of Bariatric Endoscopy, Center for Advanced \\ Endoscopy, Beth Israel Deaconess Medical Center, Harvard Medical School, 330 Brookline Avenue, Boston, MA 02115, USA. \\ E-mail: jmcohen@bidmc.harvard.edu
}

How to cite this article: Mlabasati J, Bilal M, Cohen J. Early lessons on assembling a center for bariatric endoscopy. Miniinvasive Surg 2020;4:42. http://dx.doi.org/10.20517/2574-1225.2020.32

Received: 27 Mar 2020 First Decision: 15 Apr 2020 Revised: 27 May 2020 Accepted: 11 Jun 2020 Published: 12 Jul 2020

Academic Editor: Wah Yang Copy Editor: Cai-Hong Wang Production Editor: Tian Zhang

\begin{abstract}
As the obesity epidemic continues to grow, the need for effective management strategies is more important than ever. There are several medical, endoscopic, and surgical management options available. The last decade has seen a rise in endoscopic bariatric interventions. These minimally invasive therapies can be used for patients who do not qualify or are unwilling to undergo bariatric surgery. Currently, there is limited formal training in bariatric endoscopy. In this commentary, we discuss our experience in establishing a center for bariatric endoscopy at a large academic medical center.
\end{abstract}

Keywords: Obesity, bariatric endoscopy, training, endoscopic sleeve gastroplasty

\section{INTRODUCTION}

As the prevalence and global burden of obesity continue to rise worldwide, there is a growing need for evidence-based interventions to address this issue ${ }^{[1]}$. There are a multitude of adverse health consequences associated with obesity, such as hypertension, diabetes mellitus, dyslipidemia, hepatic steatosis, some cancers, and an all-cause cardiovascular mortality ${ }^{[2]}$. The economic burden of obesity is estimated to cost approximately two trillion dollars annually ${ }^{[3]}$.

The mainstay of obesity treatment includes lifestyle modifications, pharmacotherapy, and bariatric surgery. While pharmacologic therapy has demonstrated 5\% to $10 \%$ weight loss compared to placebo, these effects are relatively modest and tend to be short-lived. Although bariatric surgery has been shown to be effective in achieving long-term weight loss, in countries following National Institute of Health criteria, it is

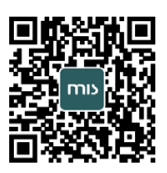


Institutional financial backing of the baratric center

Multidisciplinary team effort including gastroentrologist, bariatric surgeons, gastrointestinal radiologists, nurses, behavioral psychologists as well as registered dieticians

Robust endoscopic training in numerous endoscopic tencniques with surgical backup on hand

Minimize barriers for short and long term follow up for procedural complications

currently reserved for patients with a BMI of 40 or greater or those with a BMI of 35 or greater with obesity associated comorbid conditions. Only $1 \%$ of morbidly obese individuals undergo bariatric surgery ${ }^{[4]}$. Some factors related to this include patients fear of complications, financial constraints, and long term postbariatric surgery syndromes ${ }^{[5]}$.

Over the past decade, this has paved the way for numerous innovations in endoscopic bariatric therapies. These non-surgical therapies include intragastric balloons, endoscopic sleeve gastroplasty, gastrointestinal bypass sleeves and aspiration devices as well as other novel devices ${ }^{[6]}$. These minimally invasive therapies can be used for patients who do not qualify or are unwilling to undergo bariatric surgery. Currently, there is extremely limited formal training in bariatric endoscopy. In this commentary, we discuss our experience in establishing a center for bariatric endoscopy at a large academic medical center.

\section{STARTING A PROGRAM}

\section{Training and team}

Creating an effective endo-bariatric center requires a truly interdisciplinary team effort [Table 1]. This multidisciplinary team includes bariatric endoscopists (gastroenterologists in our center), bariatric surgeons, gastrointestinal radiologists, nurses, behavioral psychologists as well as registered dieticians ${ }^{[7]}$. This team should have a comprehensive understanding of the pathophysiology of obesity in addition to the mastery of endoluminal device and procedure specific knowledge with respect to the mechanism of action and possible complications. A comprehensive and cohesive team allows for the successful utilization of the different endoluminal therapies that may be appropriate for different patient sub-populations in achieving long term weight loss. This is also helpful in the minimization and effective troubleshooting of postprocedural complications that may arise.

While many endoscopic bariatric therapies are extensions of the current endoscopic skills gastroenterologist use daily, a bariatric endoscopist should ideally be trained in many complex endoscopic techniques including endoscopic suturing and luminal stenting. At our program we provide a strong foundation and incorporate formal didactic lectures on primary obesity therapy management [i.e., intragastric balloon (IGB), endoscopic sleeve gastroplasty (ESG)] as well as managing complications of bariatric surgery and weight regain (transoral outlet revision). We also require a minimum of $10 \mathrm{~h}$ of wet lab training followed by exposure to basic uses of endoscopic suturing (defect closure, stent fixation, fistula closure) of $>5$ in number prior to assisting on their first ESG. Observing several ESG cases prior to trainees assisting with a hands-on role is also critical. We additionally believe that the first independent 5 ESG cases be proctored. While we do not have a set number of procedures trainees are required to complete, at present they are exposed to approximately over 400 ERCP and 400 EUS procedures per year. Lastly, to help with the implementation of bariatric endoscopy in clinical practice the American Society for Gastrointestinal Endoscopy (ASGE ${ }^{[8]}$ has published a position paper to help provide guidance on the effective utilization of these therapies in clinical practice. It is important to recognize that this is just based on our early experience, and training in bariatric endoscopy can vary at each institution, depending on the endoscopist's and center's experience in training in bariatric endoscopy.

\section{Equipment}

Prior to starting a bariatric endoscopy program, it is essential to have all equipment that may be needed. A successful bariatric endoscopy program should offer patients multiple treatment options. These include 
intra-gastric balloons, endoscopic sleeve gastroplasty (ESG) procedure as well as the ability to manage post-bariatric surgery complications.

The endoscopic IGB was first proposed in the $1980 \mathrm{~s}^{[9]}$. The most used IGB is the Orbera IGB system (Apollo Endosurgery, Inc., Austin, TX, USA). ESG is the most offered endoscopic irreversible bariatric procedure. This procedure is performed with the use of an endoscopic suture system. The most widely used and available system is the OverStitch system (Apollo Endosurgery, Inc., Austin, TX, USA). The POSE procedure requires the incisionless operating platform (USGI Medical, Inc., San Clemente, CA, USA) for performing the procedure. We suggest that it is essential to have the commitment of the division and institutional leadership to provide financial support to have all available equipment prior to starting a bariatric endoscopy program.

\section{Process of setting up a team and patient recruitment}

Optimal patient selection is crucial in optimizing clinical outcomes. Patients that are referred to our center are usually BMI > 30 patients that are not candidates for bariatric surgery or a looking for minimally invasive procedures due to fear of surgical complications or long term post-bariatric surgery syndromes. All patients meet with our multidisciplinary team and undergo a comprehensive evaluation and education regarding the different endoluminal therapies available tailoring for the patient's ultimate goals. Lastly, meeting with a dietitian before and after the procedure is crucial in educating patients of the long lifestyle changes required to make durable long-lasting changes. Our program offers monthly nursing and dietitian visits as part of our comprehensive 1-year care program. Establishing a self-pay price from a large institution can take time and best to begin these conversations early with the administration with value analysis planning. Lastly, given the need for more long-term data in patients underwent bariatric and metabolic endoscopy, we also suggest developing infrastructure to carry out research studies.

\section{TYPES OF PROGRAMS}

\section{Academic medical center vs. community practice}

While a majority of the new bariatric and metabolic endoscopy therapies are being performed at large tertiary referral academic medical centers, there has been a growing amount of these procedures being performed in smaller community hospitals. Not only are some of these procedures technically feasible in the outpatient setting such as endoscopic sleeve gastroplasty with similar procedure times, they also have parallel clinical outcomes with respect to percentage of total and excess body weight loss ${ }^{[10]}$. While the clinical outcomes are similar, some challenges that community gastroenterologist will encounter in its widespread implementation is the reluctance on the part of payors to cover new procedures mentioned above as well as the infrastructure required. As the field of bariatric endoscopy continues to evolve and more studies show durable clinical outcomes with favorable safety profiles, we will see increased adoption of these procedures in the outpatient setting.

\section{Training of the endoscopist}

Given that there are limited formal bariatric endoscopy training programs available, the training of endoscopist can be challenging ${ }^{[11]}$. To undertake bariatric endoscopy as an integral part of your practice, needs long-term commitment by the endoscopist and self-driven training. Prior to performing the first case in a human, it is advisable to practice on mechanical and ex-vivo simulators. Animal laboratories can often be set-up with the help of companies manufacturing endoscopic bariatric devices ${ }^{[10]}$. In addition, there are several courses sponsored by gastroenterological organizations such as the ASGE. After basic understanding of bariatric endoscopic procedures and post-bariatric surgery anatomy is obtained, we suggest in-person shadowing at a high-volume bariatric endoscopy center. This includes shadowing the bariatric endoscopist in the office as well as during endoscopy. This allows first-hand experience of observing intra-procedural challenges and trouble shooting. In addition, for programs starting their bariatric endoscopy training 
program, it is important to ensure that nurses or assistants (endoscopy technicians) undergo a robust training session on the use of bariatric endoscopy devices. We suggest developing a pre, intra and post procedural checklist to ensure the procedures go smoothly for beginning bariatric endoscopists. When the first case is being performed, it is essential to disclose this fully to the patient. In regard to preparation for the first in-human ESG for the endoscopist, it is advisable for the endoscopist to have no other cases for that day. The schedule should be fully blocked for this case. The endoscopist should have no other clinical responsibilities such as being on-call or covering the inpatient procedures. This way the endoscopist is completely focused on this procedure alone. Discussion should be done in advance with the endoscopy team as well as anesthesia, and expectations clearly laid out that given that this will be the first procedure of the endoscopist, it could take more time. The bariatric surgery team should be available for back-up in case of any complication. Proctoring from expert clinicians is highly recommended for the first several ESG cases and beyond those given a lengthy learning curve, ongoing industry presence during ESG cases is essential. These efforts ensure that the procedure will be carried as safely as possible.

\section{CONCLUSION}

As bariatric endoscopy gains market traction, more formal training will become widely available. However, in the interim as new programs continue to develop, it is important to have a multidisciplinary approach in treating obesity. All stakeholders involved should be on board prior to starting a bariatric endoscopy program. The bariatric endoscopist should be adequately trained in not only performing basic bariatric endoscopic procedures but should be adept in managing post bariatric surgery complications.

\section{DECLARATIONS}

\section{Authors' contributions}

Conceived, drafted, edited and revised the mansucript: Mlabasati J, Bilal M, Cohen J

\section{Availability of data and materials}

Not applicable.

\section{Financial support and sponsorship}

None.

\section{Conflicts of interest}

All authors declared that there are no conflicts of interest.

\section{Ethical approval and consent to participate}

Not applicable.

\section{Consent for publication}

Not applicable.

\section{Copyright}

(c) The Author(s) 2020.

\section{REFERENCES}

1. GBD 2015 Obesity Collaborators, Afshin A, Forouzanfar MH, Reitsma MB, Sur P, et al. Health effects of overweight and obesity in 195 countries over 25 years. N Engl J Med 2017;377:13-27.

2. Guh DP, Zhang W, Bansback N, Amarsi Z, Birmingham CL, et al. The incidence of co-morbidities related to obesity and overweight: a systematic review and meta-analysis. BMC Public Health 2009;9:88.

3. Dobbs R, Sawers C, Thompson F, Manyika J, Woetzel J, et al. Overcoming obesity: an initial economic assessment. A discussion paper 
by the McKinsey Global Institute. Available from: http://www.mckinsey.com/industries/healthcare-systems-and-services/our-insights/ how-the-world-could-better-fight-obesity [Last accessed on 23 Jun 2020]

4. Deitel M. A brief history of bariatric surgery to the present. J Obes Eating Dis 2017; doi: 10.21767/2471-8203-C1-001.

5. Ju T, Rivas L, Arnott S, Olafson S, Whitlock A, et al. Barriers to bariatric surgery: factors influencing progression to bariatric surgery in a U.S. metropolitan area. Surg Obes Relat Dis 2019;15:261-8.

6. Glass J, Chaudhry A, Zeeshan MS, Ramzan Z. New era: endoscopic treatment options in obesity - a paradigm shift. World J Gastroenterol 2019;25:4567-79.

7. Shah SL, Aronne LJ, Sharaiha RZ. Setting up an endobariatric weight loss program. Am J Gastroenterol 2018;113:1567-9.

8. Sullivan S, Kumar N, Edmundowicz SA, Dayyeh BKA, Jonnalagadda SS, et al. ASGE position statement on endoscopic bariatric therapies in clinical practice. Gastrointest Endosc 2015;82:767-72.

9. Elsborg L. A novel device for removal of the gastric remnant after intragastric balloon implantation for obesity. Endoscopy 1989;21:7980.

10. James TW, Reddy S, Vulpis T, Mcgowan CE. Endoscopic sleeve gastroplasty is feasible, safe, and effective in a non-academic setting: short-term outcomes from a community gastroenterology practice. Obes Surg 2019;30:1404-9.

11. Jirapinyo P, Thompson C. How to incorporate bariatric training into your fellowship program. Gastroenterology 2019;157:9-13. 